REVISTA ANDALUZA DE ANTROPOLOGÍA.

NÚMERO 11: TRABAJO Y CULTURAS DEL TRABAJO EN LA GLOBALIDAD HEGEMÓNICA

SEPTIEMBRE DE 2016

ISSN 174-6796

[pp. 174-198]

http://dx.doi.org/10.12795/RAA.2016.11.08

Recibido: 06/06/2016

Aceptado: 29/06/2016

\title{
DESLOCALIZACIÓN DE SERVICIOS Y ACCIÓN SINDICAL: LA ORGANIZACIÓN GREMIAL DE LOS TRABAJADORES DE LOS CENTROS DE LLAMADAS DE LA ARGENTINA
}

\section{OFFSHORING SERVICE AND TRADE UNION ACTION: THE UNION ORGANIZATION OF CALL CENTERS WORKERS IN ARGENTINA}

\section{Andrea del Bono}

Consejo Nacional de Investigaciones Científicas y Técnicas (CONICET_sede ICSyAUNAJ)

\section{Resumen.}

El objetivo de este artículo es plantear una reflexión sobre los alcances y los límites de la acción sindical en el marco de procesos de deslocalización internacional y de relocalización de actividades. La idea rectora del trabajo es que las normas que regulan los mercados laborales y las que protegen la fuerza de trabajo determinan la diferencia en los procesos de deslocalización, tanto como el nivel de organización y el poder de negociación de los trabajadores. El ámbito de indagación de este texto corresponde a una actividad en la que impactan los procesos de deslocalización internacional: los servicios de atención a clientes de los centros de llamadas de la Argentina. En ese marco, se analizan las estrategias que despliegan los trabajadores para mantener las conquistas alcanzadas en el plano concreto de la relación laboral y para avanzar en la esfera de la protección de sus derechos laborales. El análisis desarrollado muestra las limitaciones de 
la acción de los sindicatos ante las permanentes reestructuraciones empresariales, y se enfatiza que la fuerza de trabajo sólo podrá recuperar poder de negociación con mayor unidad sectorial, respaldándose en estrategias que desborden las problemáticas de cada empresa particular, e incluso que traspasen las fronteras nacionales.

Palabras Clave: deslocalización internacional, sindicatos, actividades de servicios, centros de llamada.

\begin{abstract}
.
The aim of this paper is to reflect on the possibilities and the limits of trade union action under offshoring processes and relocation of activities. The guiding idea is that the difference between relocation processes are determined by the rules that governs labor markets and protects the workforce determine as well as by the level of organization and bargaining power of workers. The reserch area of this paper is an activity that is strongly impacted by the offshoring process: the customer care services of the call centers in Argentina. The issues adressed here refer to the workers strategies oriented to maintain the gains achieved in the concrete level of employment relationchips and to advance on the field of labor rights. Broadly, the analysis shows the deeper challenges of the unión action to adress the permanent corporate restructurings, emphasizing that the workforce can only regain bargaining power with greater unit at the sectorial level. This implies supported on strategies that go beyond the problems of each individual company and even beyond national borders.
\end{abstract}

Keywords: offshoring, unions, service activities, call centers.

\title{
1. INTRODUCCIÓN
}

Aunquela transnacionalización dela producción y de los flujos económicos son fenómenos claves de la dinámica capitalista largamente estudiados, hoy en día se vuelve a reflexionar sobre el tema porque sus alcances se han visto replanteados. En el escenario actual de globalización económica, la problemática de la deslocalización internacional, entendida de manera general como el traslado de los establecimientos industriales pertenecientes a determinadas firmas de unos países a otros, se aborda comúnmente desde la óptica de los países centrales, a raíz de la magnitud que adquieren sus efectos adversos en términos económicos y sociales. A la inversa, desde los países menos desarrollados, receptores de estas dinámicas, se tienden a enfatizar sus ventajas en términos de mejoras en el tejido productivo y de creación de empleos.

Según señala la literatura especializada (de la Cámara Arilla y Puig Gómez, 2007; Ermida Uriarte 2009; Flores Sanchez, 2010), estas controversias no siempre son 
realmente conducentes, con demasiada frecuencia la deslocalización se asocia con procesos económicos diferentes y de distinta naturaleza y eso complica la compresión del fenómeno, el estudio de las causas que lo impulsan, y hasta el análisis de sus consecuencias. Debido a esto, es preciso comprender con mayor precisión el fenómeno, describir sus características en ciertos ámbitos concretos, y clarificar sus posibles efectos. Con este propósito, en este texto planteamos un abordaje sobre un aspecto bastante específico y acotado, nos preguntamos por los desafíos que se plantean a los trabajadores y a sus organizaciones gremiales, en escenarios productivos fuertemente atravesados por estrategias de deslocalización de actividades, para mantener una posición firme, a partir de la cual negociar distintos aspectos, y especialmente, los que están relacionadas con las condiciones de trabajo y las relaciones laborales.

Tomamos como ámbito de indagación una actividad que consideramos emblemática de las transformaciones productivas en curso, que en los últimos años ha sido una gran receptora de los procesos de deslocalización internacional: los servicios de atención a clientes de los centros de llamadas -call centers-.

Para organizar el abordaje del estudio de caso, procuramos no perder de vista que era necesario tener en cuenta las presiones y evoluciones de los mercados globalizados pero también el peso de los actores y de los factores institucionales para incidir en esos procesos. En este sentido, una idea rectora del trabajo es que, las normas que regulan los mercados laborales y las que protegen la fuerza de trabajo determinarán la diferencia en los procesos de deslocalización, tanto como el nivel de organización y el poder de negociación de los trabajadores. Tal perspectiva conlleva el distanciamiento de las lecturas de sesgo determinista y de los análisis esquemáticos que reconocen un único ritmo y una única lógica a la globalización económica sin llegar a dar cuenta de las diferentes lógicas y ritmos existentes según sean los distintos mercados analizados.

En el marco de esta problemática, planteamos como cuestión central la reflexión sobre las estrategias que despliegan los trabajadores de los centros de llamadas para mantener las conquistas alcanzadas en el plano concreto de la relación laboral y para avanzar en la esfera de la regulación institucional, protegiendo los derechos laborales en un contexto productivo cambiante, amenazado por el problema de la deslocalización.

En términos metodológicos el trabajo investigativo realizado se basó en un exhaustivo análisis de fuentes documentales (prensa periódica, páginas web, boletines electrónicos de cámaras empresariales y bibliografía especializada) y en una veintena de entrevistas semiestructuradas a trabajadores de centros de llamadas y militantes sindicales y de base (a mediados de 2010, realizamos trabajo de campo en la ciudad de Córdoba, entre las entrevistas que más utilizamos en este texto se destacan las realizadas a referentes de las nuevas organizaciones de trabajadores de los centros de llamadas: el Sindicato 
de Teleoperadores, la Asociación de Trabajadores Argentinos de Centros de Contacto (ATACC), la Coordinadora de Trabajadores de Call Centers -La Coordinadora- y el colectivo Calls en Lucha). Asimismo, entre 2009 y 2010, realizamos una docena de entrevistas semiestructuradas a gerentes de Recursos Humanos de las multinacionales más importantes que operan en la Argentina en el segmento de exportación se servicios empresariales, con el objetivo de llegar a conocer la dinámica de las operaciones offshore.

El texto se organiza en seis apartados. El primero presenta algunas de las cuestiones conceptuales que permiten acotar el fenómeno de las deslocalizaciones, vinculándolo con el problema que plantean para la acción sindical; el segundo presenta la relevancia actual de los servicios empresariales a distancia, sitando en ese marco, a los sercios de atención a clientes; en el tercero se presentan los rasgos más salientes de la evolución de los centros de llamadas en la Argentina; en el apartado siguiente se analizan las dinámicas de relocalización y deslocalización que impulsan el crecimiento de los centros de y la incidencia de dichas dinámicas en el trabajo, los salarios y las condiciones de trabajo de los agentes telefónicos, en los dos últimos apartados se describen detenidamente la forma que adopta la organización colectiva de los trabajadores de los centros de llamadas y el papel que juegan las organizaciones sindicales, en ambos casos, se analizan el alcance y las limitaciones de las respuestas de los trabajadores frente a los problemas que les plantean en el plano cotidiano de las relaciones laborales el sostenido proceso de relocalizacióndeslocalización que impulsa el desarrollo de la actividad.

\section{GLOBALIZACIÓN, DESLOCALIZACIÓN Y ACCIÓN SINDICAL, NOTAS PARA LA DISCUSIÓN}

El fenómeno de la globalización es el telón de fondo sobre el que discurren las tendencias más emblemáticas de la reestructuración productiva contemporánea, entre éstas, la deslocalización internacional. Este fenómeno se caracteriza por la expansión y la profundización de la producción y de la competencia global sin trabas y ha sido motorizada, -por destacar algunos de sus factores más potentes y novedosos-, por el desarrollo de las tecnologías de la información y la comunicación -en particular las redes digitales- por la reducción de los costos del transporte y por la liberalización del comercio y la inversión extranjera directa; todo lo cual ha brindado condiciones inusitadas para fragmentar el proceso productivo contando con la circulación electrónica de determinados productos (software) y la contratación de servicios fuera de las fronteras del país en el que se demandan. Como señala Harvey (1998), en la etapa actual de capitalismo las nuevas tecnologías permiten comprimir el tiempo y el espacio y ampliar los márgenes del beneficio gracias a que la especialización ya no requiere de cercanía geográfica.

En la actualidad, las dinámicas deslocalizadoras admiten una gran variedad de formas y contenidos, que por razones de espacio no analizaremos en esta oportunidad. Tomamos 
en este texto el fenómeno en su acepción más amplia, que refiere al proceso que permite a las empresas desplazar hacia una ubicación transfronteriza sus fuentes de suministro o sus capacidades productivas. Esta acepción, abarca los casos más claros de cierre de empresas y de fábricas que migran hacia el extranjero y otras situaciones, más complejas y de contornos imprecisos, que se constituyen igualmente en una fuente de incertidumbre para los trabajadores (Flores Sánchez, 2010; de la Cámara Arilla y Puig Gómez, 2007).

Desde el punto de vista social, los efectos de la deslocalización internacional se analizan en el plano de la creación y destrucción de empleos, en relación con la calidad del trabajo y en función de las relaciones laborales que auspician o clausuran las dinámicas de deslocalización.

Una primera lectura permitiría suponer, como apunta Luengo (2010), que el análisis y valoración de los procesos de deslocalización es diferente según se considere desde los países que originan el traslado de las actividades productivas o desde los países de destino de las mismas. En el primer caso, es de esperar que las miradas sean por lo menos escépticas ante la pérdida de establecimientos productivos y de puestos de trabajo que ocasionan estos desplazamientos. En cambio, los efectos de la relocalización son generalmente considerados como positivos en los países receptores, ya que comúnmente permiten desarrollar sus economías, transformar en cierta medida sus sistemas productivos, a veces con la introducción de actividades previamente inexistentes, mejorar los niveles salariales, etc. No obstante, esta perspectiva se convierte en preocupación cuando una parte de las capacidades productivas deslocalizadas buscan nuevos emplazamientos; o también cuando las empresas locales diseñan estrategias de internacionalización que suponen el cierre de plantas y destrucción de empleos. Al respecto, tal como sostiene Ermida Uriarte (2007), parece difícil esperar que en el largo plazo se sostenga un juego de relaciones laborales relativamente avanzado en el país de destino, ya que este mismo efecto puede arriesgar una nueva deslocalización desde allí hacia otro lugar.

En relación con los efectos de las dinámicas de deslocalización, resulta incuestionable la tendencia hacia la amplitud y diversificación de los impactos de las relocalizaciones sobre los puestos de trabajo (Flores Sánchez, 2010). Desde este ángulo, no serían los efectos sobre los niveles del empleo los más significativos, los mayores impactos parecen corresponderse con el plano de las relaciones laborales y del trabajo concreto, a partir del aumento de la vulnerabilidad de los trabajadores y de las consecuencias que dicha debilidad acarrea en el plano de la negociación de las condiciones de trabajo.

Este proceso plantea enormes desafíos a la acción sindical, frente a la creciente movilización del capital, que se articula cada vez más en el espacio transnacional, se reduce el margen de intervención y de presión de los trabajadores (Peck 1990). Estas tendencias estarían erosionando el poder de negociación de los trabajadores por una doble vía, desde arriba, debido a la movilidad actual y potencial del capital vis a vis la del trabajo y desde abajo, 
cuando las condiciones sociales y las normas regulatorias locales son desmanteladas en los intentos por exponer el trabajo al mercado y por atraer o retener al capital móvil.

En el caso de América Latina, haciendo extensiva esta situación a la Argentina, el problema no es tanto cómo enfrentar o evitar deslocalizaciones, sino cómo lucir atractivos ante eventuales inversores dispuestos a localizarse. El verdadero problema se presenta entonces, como ha ironizado Ermida Uriarte, “cuando comenzamos a competir entre nosotros a ver quién es más seductor y quién obtiene los favores de esa dama - o ese caballero-, según el caso" (2007:10).

\section{EL CAMBIO EN LA LOCALIZACIÓN INTERNACIONAL DE LOS SERVICIOS EMPRESARIALES}

En el marco de las tendencias mencionadas, los empleos del sector servicios relacionados con las tecnologías de la información y la comunicación se han visto afectados sostenidamente por las decisiones de las empresas de externalizar y deslocalizar tareas y funciones consideradas accesorias. Como acabamos de sugerir, esta es una cuestión que afecta a los trabajadores de los países centrales pero que contribuye, también, a cambiar radicalmente los mercados laborales y las condiciones de trabajo de los países y las regiones con menor grado de desarrollo (Sassen, 2003; 2007). Principalmente, los sectores involucrados en la externalización y la deslocalización internacional son los bancos, las compañías de seguros y de telecomunicaciones, los proveedores de atención de salud y, profundizando la tendencia de las últimas décadas, la producción fabril. Impulsada por dicha evolución, la actividad de los centros de llamadas es una de las formas de trabajo más extendida y uno de los sectores con mayor capacidad de creación de empleos del sector servicios, a nivel mundial.

Asimismo, en este contexto, la comercialiación de "servicios empresariales a distancia" ha adquirido una creciente importancia económica; este tipo de provisión de servicios consiste en la prestación a distancia de una serie de actividades o funciones de carácter no esencial, como las tareas administrativas periódicas o rutinarias, la asistencia técnica, los servicios de apoyo logístico, la atención al consumidor o los servicios post venta. Un elemento característico es que la provisión del servicio se realiza bajo la modalidad de comercio transfronterizo: el servicio mismo "cruza la frontera" y el usuario del servicio importado puede recibirlo por medio de su sistema de telecomunicaciones o por correo (Piña, 2005:14).

A escala global, dicha evolución se corresponde con las tendencias de la economía mundializada que marcan un rápido crecimiento del mercado offshore de servicios debido a la profundización del alcance de las estrategias de "tercerización de procesos de negocios" (ATKearney, 2007) y con la consolidación de proveedores especializados que operan desde localizaciones geográficas que presentan ventajas comparativas. 
A continuación, presentamos sintéticamente algunos rasgos de la evolución de los centros de llamadas en la Argentina para contextualizar el análisis posterior sobre la dinámica que adopta la acción sindical en el sector.

\section{CENTROS DE LLAMADAS Y COMERCIALIZACIÓN DE SERVICIOS: TENDENCIAS DE EVOLUCIÓN}

Durante la última década se consolidaron en la Argentina un grupo de empresas especializadas en la tercerización de procesos de negocio, el crecimiento sostenido de las operaciones de los centros de llamadas y de las empresas comercializadoras de servicios (servicios en mercadeo, cobranza, soporte y servicio al cliente, entre otros) que gestionan sus operaciones, son un reflejo de esa situación. A partir de 2002, con la salida de la convertibilidad monetaria y la devaluación del peso frente al dólar, el país se convirtió en una de las localizaciones de América Latina con mejores condiciones para el desarrollo de un mercado de centros de llamadas.

Con condiciones favorables, las empresas comercializadoras de servicios aceleraron su expansión y asumieron la gestión de información y procesos de negocio que con anterioridad gestionaban directamente las mismas compañías que se transformaron en sus grandes clientes: bancos, operadores turísticos, operadoras de telecomunicaciones, empresas de software y servicios informáticos, entre otros sectores y actividades.

Desde los centros de llamadas de la Argentina, se comenzó a brindar servicios de teleatención a usuarios ubicados en Estados Unidos y en España (principales destinos del sector de servicios y de tercerización de procesos de negocio), y en otros países de la región. En base a esta actividad se formó un sector importante en términos de generación de empleos, mayormente registrados y formales, que posibilitó el ingreso al mercado laboral de miles de trabajadores jóvenes.

Durante la etapa de mayor expansión, desde 2002 y hasta finales de 2008, las diez empresas de comercialización de servicios más importantes del país eran compañías filiales o subsidiarias de grupos multinacionales, nucleadas en un sector dominado por la inversión extranjera directa, y caracterizado por la concentración de las operaciones. Estas firmas se impusieron en el mercado local y lideraron la expansión del mercado de offshoring de servicios (Del Bono, 2010). ${ }^{1}$

1. Más allá de las fusiones y adquisiciones que se han registrado desde 2002 hasta la actualidad, la conformación del sector de telemarketing sigue estando hegemonizada por grandes firmas internacionales. Mencionamos a continuación, las empresas de telemarketing, según información actualizada en el 2015 por la Cámara Argentina de Centros de Contacto: Aegis Argentina; Allus Argentina; Apex America; Arvato; Atento; Cat Technologies Argentina S.A; Clienting Group S.A.; Hycite; Indicom SA; Multiconex; Next; Plus SA; PYD; Task Solutions; Tecnovoz; Telemercado; Teleperformance; TeleTech; V/N Global BPO; Voicenter SA; Xerox. 
Según la información publicada por la Cámara Argentina de Centros de Contacto (CACC), el sector llegó a su punto más alto de expansión durante 2008 con la generación de 70.000 empleos, superando ampliamente los 45.000 de finales de 2006 (CACC, 2013). Durante 2007, las exportaciones de servicios representaron un $38 \%$ sobre el total facturado, alcanzando uno de los picos más altos de la etapa de expansión (Frost and Sullivan, 2008). A finales del 2008, con el impacto de la crisis económica global, las exportaciones de servicios mostraron por primera vez una tendencia decreciente; este cambio marcó el inicio de un nuevo ciclo para las empresas comercializadoras de servicios de teleatención que reorientaron sus operaciones hacia el mercado interno, durante ese año las operaciones offshore descendieron al 33\% de la facturación total del sector (Convergencia 2008).

De allí en más, esta tendencia de retroceso de las exportaciones de servicios se profundizó aceleradamente. Según los últimos datos sectoriales publicados por la Cámara empresarial, a mediados de 2013, el peso de la facturación offshore representó tan sólo el 5\% de la facturación total del sector (CACC, 2013). El impacto de la reorientación de actividades de los centros de llamadas hacia el mercado interno se refleja en el dato de creación de empleo, que descendió del punto más alto de 70.000 empleos generados durante 2008, a 56.200 a finales de 2012 (CACC, 2013).

Del análisis de esta evolución surge nuestra pregunta sobre las estrategias que impulsaron los propios trabajadores y sus representaciones sindicales frente a la pérdida de empleos y a la fragmentación que conlleva, para el conjunto de la actividad, la dinámica de relocalización y deslocalización internacional. Antes de responder ese interrogante, vamos a mencionar brevemente algunos de los factores que explican las tendencias que acabamos de mencionar.

En primer lugar, hay que señalar que las empresas comercializadoras de servicios de teleatención dejaron de encontrar en la Argentina las condiciones extremadamente favorables que tuvieron a la salida de la crisis económica del año 2001, puntualmente, el país ofrecía por ese entonces los salarios comparativamente más bajos de toda América Latina (ATKearney, 2007), niveles históricos de desocupación, y una fuerza de trabajo compuesta por jóvenes con buenos niveles de cualificación y con mucha necesidad de trabajar. En el escenario de recuperación económica que se inició en 2003, se produjo una paulatina apreciación del peso frente al dólar, el mejoramiento general del nivel de salarios de la economía en su conjunto, y avances muy significativos en términos de protección de derechos laborales y de creación de empleos genuinos. La conjunción de estos elementos con las consecuencias macroeconómicas de las crisis de 2008, puso un freno a la localización de operaciones offshore en la Argentina, al tiempo que alentó la relocalización de los centros de llamadas en el interior del país y la deslocalización 
internacional hacia otros países de la región con costos salariales más bajos y relaciones laborales más flexibles.-

En segundo lugar, con esas relocalizaciones las empresas consiguieron reducir sus costos y escapar al proceso de saturación de los centros urbanos -Buenos Aires, Córdoba- donde la competencia por la mano de obra y los altos índices de rotación de los agentes telefónicos se traducían en escasez de recursos humanos. Asimismo, provincias argentinas como Tucumán, San Luis y Chacho, impulsaron la radicación de centros de llamadas mediante el desembolso de abultados subsidios con el objetivo de impulsar la creación de nuevos empleos para las frágiles economías regionales (Del Bono, 2013).

A partir de 2008, los Estados provinciales tuvieron un rol clave (Moench, 2011), lideraron las estrategias regulatorias y las orientaron hacia la atracción de las empresas de telemarketing por medio de diversas ventajas otorgadas para que los centros de llamadas se instalasen en su territorio: exención tributaria, subsidios por puesto de trabajo subsidios parciales en los salarios de los primeros años de actividad-, bonificación de cargas laborales, capacitación de personal, donación de infraestructura edilicia y aportes para equipamiento y oficinas. Desde entonces, se generó un verdadero efecto "cascada" entre provincias argentinas que comenzaron una verdadera competencia por "facilidades", en términos de la legislación ofrecida a las empresas comercializadoras de servicios. Esto explica el proceso de relocalización masiva de centros de llamadas en el interior de la Argentina. Según datos de la Cámara Argentina de Centros de Contacto, el 69\% del empleo del sector está radicado en el interior del país (CACC. 2013).

Finalmente, esta misma dinámica impulsó la deslocalización internacional de los servicios de teleatención que se prestaban desde la Argentina, a otros países de la región. En los últimos años la región andina conformada por Colombia y Perú, superaron a los mercados que conforman el cono sur (Chile, Argentina, Paraguay, Uruguay y Bolivia), no sólo en el volumen de las operaciones sino también en la generación de puestos de trabajo. En la actualidad, Centroamérica y el Caribe, son considerados los nuevos polos de servicios offshore (Pron-Perú: 2015), donde han comenzado a instalarse las grandes empresas multinacionales especializadas en la comercialización de servicios.

En términos de salarios y condiciones de trabajo, las empresas aseguran así su competitividad y los bajos costos mientras que los trabajadores ven degradarse sus condiciones laborales.

\section{SALARIOS, JORNADA Y CONDICIONES DE TRABAJO: LAS VARIABLES DE FLEXIBILIZACIÓN Y “AJUSTE”}

Como han analizado Knorringa y Pegler (2006), las grandes firmas tercerizadoras que gestionan servicios de teleatención ubican a sus agentes telefónicos en plataformas de trabajo, baratas o poco costosas, de tal manera que la relocalización facilita la segregación 
de los mercados internos de trabajo, limitando la oportunidad de los trabajadores de conseguir buenos empleos, bien remunerados. Así se aprovechan ventajas comparativas derivadas de la segmentación del mercado de trabajo en función del lugar escogido para la localización de los centros de llamada: los trabajos menos calificados como el de atención a clientes, el de los data entry, y el telemarketing de menor valor agregado, son segregados y relocalizados en zonas suburbanas u offshore con costes más bajos (Holman; Batt; Holtgrewe, 2007). En este contexto, la organización del trabajo; de la jornada laboral; la gestión de los salarios; de los contratos y de las propias relaciones laborales, son variables que los centros de llamada "ajustan" permanentemente en función de sus metas.

A continuación, presentamos de manera sintética cómo se regulan dichos factores en el caso argentino ya que es, en ese terreno, en el que se plantea el desafío para la acción sindical, precisamente, en el plano de la regulación de los salarios y de la jornada laboral, e incluso, de las propias condiciones de trabajo.

Entre 2002 y 2008, etapa de mayor expansión de los centros de llamadas en la Argentina, las empresas comercializadoras recurrieron mayoritariamente para la conformación de sus plantillas a contratos laborales formales, -por tiempo indeterminado, según regula la legislación laboral argentina-, ajustándose además a ciertas políticas de responsabilidad social. Los contratos formales se mantuvieron a partir de 2008, cuando la actividad se reorientó casi exclusivamente al mercado doméstico.

Para los trabajadores y trabajadoras jóvenes, la naturaleza formal del contrato que ofrecen los centros de llamadas es el principal motivo por el que buscan ingresar al sector. Según un relevamiento publicado recientemente, para el 34\% de los empleados del sector, el trabajo en un centro de llamadas constituye la primera experiencia laboral formal (CACC, 2013). En este sentido, y tal como hemos analizado en oportunidades anteriores (Del Bono, 2013; 2008), las contrataciones informales no son la norma en las grandes empresas del sector, sin embargo, como veremos a continuación, este factor no cancela la existencia de otras situaciones de flexibilización laboral y salarial, que auspician procesos de precarización.

En la Argentina, la fuerza de trabajo de los centros de llamadas presenta trayectorias laborales típicamente juveniles que transitan por el circuito de trabajos "para jóvenes" -cadenas de comida rápida, cines, vendedores/as de centros comerciales, servicios de mensajería, etcétera-. Mayoritariamente, se trata de una población joven que trabaja y estudia; según la Cámara Empresarial, el 76 \% de la población de trabajadores de los centros de llamadas son jóvenes de entre 18 y 30 años, entre ellos, un $80 \%$ tiene estudios secundarios completos, y un $44 \%$ continúa estudiando (nivel terciario y/o universitario) mientras trabaja (CACC, 2013). Al interior de los centros de llamadas, los jóvenes con mayor formación se insertan en las actividades de servicios mejor remuneradas 
que tienen requerimientos de saberes técnicos y/o conocimiento de idiomas (cuentas offshore), mientras que los jóvenes con niveles más bajos de preparación trabajan en la atención a clientes, en servicios orientados al mercado interno.

En relación a la jornada de trabajo, las empresas que gestionan servicios de teleatención aplican una política de flexibilización de la organización y del tiempo de trabajo lo que se traduce en el establecimiento de variadas estructuras de turnos de trabajo y jornadas laborales. En los centros de llamadas se recurre a contratos a tiempo parcial (4 horas), también a contratos full time de cinco días a la semana y una jornada laboral de 6 horas, y en algunos casos, hasta de ocho horas diarias, a pesar de que la jornada legal en el sector es de seis horas. Sin embargo, en estos establecimientos coexisten distintas estructuras horarias, lo cual refleja el amplio margen de flexibilidad con el que se mueven las empresas, desatendiento frecuentemente las situaciones de desgaste y estrés que son propias del trabajo de teleatención. Como veremos en el apartado siguiente, esta situación ha motivado la movilización y la protesta de los trabajadores, frente a la falta de intervención de sus propias dirigencias gremiales.

$\mathrm{Al}$ igual que las jornadas, los salarios de los centros de llamadas, también presentan una gran dispersión. El salario de los agentes telefónicos varía, en primer lugar, en función de los servicios prestados, por ejemplo, existen diferencias salariales que oscilan entre un $20 \%$ y $40 \%$, con promedio de $30 \%$, entre un agente de soporte técnico y otro de atención al cliente. Finalmente, es importante mencionar que las remuneraciones de los agentes tienen un componente variable importante, la relación de los adicionales con el salario básico de convenio oscila entre un 15\% y hasta un 40\% que se suman al sueldo base, lo que refleja el amplio margen de flexibilidad salarial con el que se gestiona la actividad.

En síntesis, las empresas que gestionan los centros de llamadas gozan de un amplio margen de libertad para organizar, con un alto grado de flexibilidad, cuestiones claves como la organización del trabajo, la extensión de la jornada laboral y el monto y composición de los salarios de los agentes telefónicos.

En un contexto productivo de esta naturaleza es importante interrogarse sobrela capacidad de los trabajadores organizados para evitar el paulatino deterior de sus condiciones de trabajo y el debilitamiento de las relaciones laborales que regulan la actividad.

\section{LA REPRESENTACIÓN SINDICAL DE LOS TRABAJADORES DE LOS CENTROS DE LLAMADAS}

En la Argentina, el período de la post Convertibilidad iniciado en 2002, no solo supuso importantes modificaciones en el contexto económico-productivo, sino también un cambio de época en lo referente al marco político-institucional de las relaciones laborales. Durante las presidencias de Néstor Kirchner (2003-2007) y de Cristina Fernández de Kirchner (2007-2015), se produjo un cambio en el rol del Estado, en tanto coordinador 
de la negociación colectiva, al pautar incrementos salariales a través de distintos institutos laborales y en tanto agente de contralor, a través de diversas medidas de inspección y control de las condiciones de trabajo- y una reorientación de la justicia laboral, que restablece algunos de los principios protectorios del trabajo (Palomino, 2007).

Estas mutaciones contribuyeron a brindar una mayor gravitación al accionar sindical, un hecho que quedó reflejado en el sostenido crecimiento de algunos indicadores claves, tales como las negociaciones colectivas, los conflictos laborales -en detrimento de los conflictos sociales predominantes en los años'90- y las tasas de afiliación (Palomino y Trajtemberg, 2007, Trajtemberg et al, 2009, Senén González y Haidar, 2009). El renovado protagonismo de los sindicatos puede interpretarse, en este contexto, como una de las manifestaciones más evidentes de un cambio de época.

En este marco, la literatura académica se ha interrogado por los reales alcances del resurgimiento del actor sindical que tuvo lugar durante esos años. Como venimos señalando, una de las cuestiones de fondo de este debate, alude a las estrategias desplegadas por los trabajadores y sus organizaciones gremiales para afrontar los retos que les plantean las políticas de deslocalización productiva y de tercerización laboral en sus diversas variantes, ya que estos procesos se han seguido reactualizando y profundizando, aún en el escenario referido de recuperación de derechos laborales, económicos y sociales, afectando a los derechos colectivos de los trabajadores y alentando tendencias renovadas de precarización laboral.

Específicamente, en este texto nuestra atención se dirige hacia una actividad que en las últimas décadas sufrió grandes transformaciones en ese sentido. En la Argentina, la evolución del sector de telecomunicaciones se vincula estrechamente con el surgimiento de la actividad de los centros de llamadas y con las condiciones laborales de sus trabajadores ya que esta nueva actividad, quedó excluida de la protección de los gremios del sector, luego de la tercerización y privatización de la operadora estatal de telecomunicaciones (Senén González y Garro, 2008). En ese proceso los trabajadores de los centros de llamadas quedaron comprendidos en el marco de una representación sindical fragmentada, y por convenios con peores condiciones generales en comparación al que rige para los trabajadores telefónicos, reduciéndose así la capacidad de negociación de los agentes que trabajan para las empresas comercializadoras de servicios.

Uno de los elementos distintivos del caso argentino estuvo dado por el carácter unilateral del diseño de las políticas de reestructuración del sector de telecomunicaciones que relegaron de las decisiones a la hasta entonces muy influyente Federación de Obreros y Empleados Telefónicos dela República Argentina(FOETRA). Asimismo, el debilitamiento sindical se acentuó con la pérdida del monopolio de la representación de los trabajadores del sector cuando, con los nuevos negocios y las obras de modernización, entraron en escena numerosas empresas subcontratistas y nuevos sindicatos como la Unión Obrera 
de la Construcción (UOCRA) y el Sindicato de Empleados de Comercio (Federación Argentina de Empleados de Comercio y Servicios) (Walter y Senén González, 2000).

Puntualmente, la representación gremial de los agentes telefónicos de los centros de llamadas quedó a cargo de la Federación de Empleados de Comercio, a través del Convenio Colectivo de Empleados de Comercio (CCT No130/75). Este encuadramiento convencional ubica a los empleados del sector en un terreno nebuloso e inespecífico en casi todos los campos, desde la definición de la duración de la jornada laboral, pasando por el control y la regulación de las condiciones y medio ambiente de trabajo, hasta llegar al terreno de las retribuciones -donde no existe una tabla de salarios unificada común para todos los trabajadores del sector- (Del Bono y Henry, 2008). Asimismo, el hecho de que la representación de los agentes telefónicos quedara hegemonizada por un gremio ajeno al sector de telecomunicaciones -el sindicato de Comercio- desembocó en la proliferación de conflictos (por re-encuadramiento) y movilizaciones (por condiciones de trabajo) que revistieron, según veremos, características novedosas.

El Sindicato de Empleados de Comercio es uno de los gremios más importantes de la Argentina y no perdió su posición de relevancia ni siquiera luego de atravesar la década de 1990, una época sumamente adversa para los sindicatos debido al impacto de la erosión de la actividad industrial, de las reformas estructurales neoliberales y del alto nivel de desempleo. En esa situación, algunos sindicatos optaron por una "supervivencia organizativa" (Murillo, 1997), en tanto eligieron negociar con el gobierno los condicionamientos de las reformas estructurales en marcha. El sindicato de Comercio estuvo dentro de este conjunto de gremios que optaron por una adaptación que consistió, básicamente, en la incursión en un conjunto de actividades empresarias de servicios sociales que las mismas reformas estructurales habían hecho surgir como, por ejemplo, la creación de administradoras de fondos de pensión o la reorganización de sus obras sociales. Esto les permitió compensar la decadencia de sus recursos económicos y ganar mayor autonomía, no sólo respecto al estado, sino también de sus propias bases. En aquel contexto el sindicato de Comercio optó por la salida más "capitalista" al reforzar su línea de provisión de servicios, adhiriendo así a un modelo de "sindicalismo de negocios" (Novick, 2001).

Por lo tanto, las empresas comercializadoras de servicios contaron en su etapa de expansión con la interlocución de un sindicato aggiornado y desprovisto de una historia de reivindicaciones y de tradición de lucha que pudiese servir de referencia para los trabajadores de los centros de llamadas. Esta es una cuestión que cobra toda su importancia si se toma en cuenta que mayoritariamente los agentes telefónicos son jóvenes que recién ingresan al mercado laboral. En el marco de una actividad con muchos aspectos cuestionables, esos trabajadores no tuvieron el respaldo de una organización sindical que incentive la organización colectiva para conformar una identidad común, a partir de la 
cual fortalecer su posición respecto a las empresas. Durante muchos años, los centros de llamadas no tuvieron cuerpo de delegados gremiales, a raíz de la inexistencia de canales de comunicación entre el sindicato y sus bases, para encausar los problemas y reclamos cotidianos. Tampoco se plantearon políticas sindicales de protección de la salud de los agentes telefónicos, a pesar de las graves patologías derivadas del trabajo de teleatención (enfermedades osteomusculares, traumatismos y trastornos de origen psíquico).

El sindicato de Comercio mantuvo además una posición conciliadora con las empresas comercializadoras de servicios. Considerando que el poder de negociación sindical se ve disminuido frente a la estrategia de deslocalización de los centros de llamadas, se descartó la confrontación en pos de la preservación de las fuentes de trabajo. En términos del que fuera Secretario de Asunto Gremiales del sindicato: "se instalan en Argentina por una cuestión de costos (...) Esto habla de la verdadera historia de por qué se instalan en Argentina, a tal punto que cuando sienten alguna presión gremial o política amenazan con irse a otros países donde los costos son mejores, como Chile o Perú. Esa es la respuesta que se obtiene y frente a eso nosotros no podemos hacer nada" ${ }^{2}$.

Esta situación de hegemonía de un sindicato negociador y sin estrategias para representar a los trabajadores y trabajadoras de los centros de llamadas comenzó a discutirse en los últimos años, a través de una serie de movilizaciones y conflictos que enfrentaron las "burocracias sindicales" que detentan la representación formal de los trabajadores planteando una nueva dinámica sindical, mucho más activa, aunque fragmentada y heterogénea. Tal como hemos analizado en, Del Bono y Bulloni (2013), el dinamismo del accionar gremial dirigido a organizar colectivamente a los trabajadores de los centros de llamadas no es atribuible entonces al accionar del sindicalismo "clásico" sino más bien al rol que asumieron nuevas organizaciones de trabajadores que interpelaron su lógica. En el apartado que sigue analizamos las estrategias desplegadas por esos grupos, tratando de caracterizarlas.

\section{LA INTERPELACIÓN A LAS FORMAS CLÁSICAS DE ORGANIZACIÓN GREMIAL: SUS ACTORES Y ESTRATEGIAS}

En la Argentina, los efectos adversos de la externalización de actividades y de la tercerización laboral vienen siendo puestos en evidencia desde hace varios años debido a la sucesión de reclamos protagonizados por empleados de empresas de servicios, proveedoras, contratistas y subcontratistas. Esas protestas revelaron la existencia de una "subclase" de trabajadores encuadrados por convenios colectivos que, contrariamente a los principios del derecho laboral, presentan condiciones laborales sensiblemente menos favorables que las derivadas de los convenios colectivos de la actividad.

2. Entrevista: Secretario de Asuntos Gremiales del Sindicato de Empleados de Comercio, noviembre 2006. 
Distintas acciones directas - por ejemplo, los paros de los trabajadores de las empresas contratistas de Metrovías (Subterráneo de Buenos Aires); los reclamos pioneros de los empleados de las distintas subcontratistas de Telefónica de Argentina y de los trabajadores de los centros de llamadas tercerizados; los cortes de vías en el ferrocarril Roca, entre otros, llamaron la atención sobre el estado de deterioro de los derechos colectivos de los trabajadores de las firmas tercerizadoras. Centralmente, los reclamos gremiales que tomaron trascendencia pública durante la última década se caracterizaron por la proliferación de controversias de representación y encuadramiento sindical, que tornaron más compleja la negociación colectiva del trabajo y la propia organización de los trabajadores.

Estas movilizaciones se inscriben en los procesos de resurgimiento de la protesta gremial de la última década, en los que la organización de los trabajadores de base adquirió un papel preponderante, disputando incluso el poder a las dirigencias gremiales, que interpretaron el proceso de revitalización del conflicto laboral en los lugares de trabajo como una amenaza a su propia hegemonía (Abal Medina et al, 2009; Lenguita, 2011; Figari et al., 2010).

En el caso de los centros de llamadas, hemos identificado un primer grupo de reclamos $\mathrm{y}$ acciones de distintos colectivos de trabajadores que se caracterizan por transitar por fuera de las estructuras sindicales tradicionales y hegemónicas y por ser el resultado de un trabajo de organización desde las bases. Experiencias de distintos colectivos, tales como el Sindicato de Teleoperadores ${ }^{3}$, que se conformó como una iniciativa de un grupo de militantes y de trabajadores afines a la Central de Trabajadores de la Argentina (CTA), que se propusieron crear un sindicato de oficio (teleoperadores) como alternativa al sindicalismo "hegemónico" y "corrompido"; agrupaciones como Calls en Luch $^{5}$ y La Coordinadora ${ }^{6}$, que sin responder ningún partido político en particular apuntaron a construir una organización de trabajadores de base sustentada en prácticas

3 Entrevista al abogado del Sindicato de Teleoperadores, realizada en Córdoba, mayo de 2010.

4. Uno de los pilares centrales del modelo sindical argentino, erigido hacia mediados del siglo pasado y que perdurara hasta nuestros días, lo constituye el instituto de la personería gremial. Según la legislación vigente, el otorgamiento por parte del Estado de esta personería única al sindicato mayoritario de la rama implica que éste dispone del monopolio de la representación en las acciones paritarias (Ley 23.551 y decreto 467/88). En la actividad de los centros de llamadas, el sindicato de Comercio, detenta esa condición de sindicato mayoritario.

5. Entrevista grupal a cinco integrantes de Calls en lucha, realizada en Córdoba, mayo de 2010.

6. Entrevista a dos dirigentes históricos de La Coordinadora de Trabajadores de Call Centers y empresas de Telefonía Celular, realizada en Córdoba, mayo de 2010. 
verdaderamente renovadas; o grupos más combativos que protagonizaron conflictos y tomas de los lugares de trabajo, como Teleperforados y Colgá la Vincha, reflejaron la conflictividad laboral existente en los centros de llamadas de Buenos Aires y Córdoba, dos de las ciudades con mayor concentración de agentes telefónicos del país.

Con matices y diferencias estas agrupaciones apuntaron, según surge del análisis de las entrevistas que realizamos a sus dirigentes, a diferenciarse del sindicalismo hegemónico representado por el sindicato de Comercio y de lo que veían como el "carácter burocrático" y "pasivo" de las estructuras gremiales. En este sentido, para estos colectivos de trabajadores de base, las características asumidas por la representación sindical de los trabajadores de los centros de llamadas eran expresión de una "estructura arcaica", pero a pesar de ello, con pocas fisuras. Por esa razón, optaron por generar acciones y prácticas desplegadas al margen de las estructuras sindicales tradicionales, entre algunas de ellas, movilizaciones y marchas de trabajadores autoconvocados; intervenciones en la vía pública para llamar la atención de la ciudadanía sobre los aspectos desgastantes y estresantes del trabajo en las plataformas de teleatención; volanteadas en la puerta de los lugares de trabajo con información para los propios compañeros de trabajo sobre sobre las desventajas del encuadramiento gremial, y una serie de actividades diversas coordinadas con organizaciones de la sociedad civil. Una característica común de estas nuevas organizaciones es la militancia y activa presencia en las redes sociales de internet. En la mayoría de los casos, se destaca la preferencia de estos colectivos por el trabajo de base en un plano muy cercano a la clandestinidad, como una estrategia de preservación frente a la manifiesta política antisindical desplegada en los centros de llamadas (Del Bono y Bulloni, 2013).

Entre los reclamos más concretos de estos colectivos de trabajadores de base hemos identificado, como objetivo prioritario, el reencuadramiento de los trabajadores del sector en el gremio telefónico, el mejoramiento de las condiciones y medio ambiente de trabajo, y en los últimos años, la aprobación de un Proyecto de Ley para regular la actividad a partir de un Estatuto para los trabajadores de los centros de llamadas. Este intento de regulación se planteó como una herramienta para combatir la atomización de los trabajadores y la heterogeneidad de las condiciones de trabajo que existen en la actividad.

Durante los años de gran expansión de las empresas comercializadoras de servicios de teleatención, las agrupaciones mencionadas contribuyeron activamente a instalar el clima de reclamos y protestas que se extendió en algunos de los centros de llamadas más importantes del país, dedicados prácticamente con exclusividad a la atención offshore. Sin embargo, no llegaron a constituirse en una alternativa a la hegemonía del sindicalismo tradicional. Con el paso del tiempo, la dificultad de expandir sus bases de adherentes y la problemática de la alta rotación que caracteriza al trabajo de los centros 
de llamadas, fueron algunos de los factores que contribuyeron a relativizar el alcance de estas experiencias.

En nuestra interpretación, estas dificultades en el plano de las relaciones colectivas de trabajo reflejan los límites que encuentra la organización de los trabajadores, en el seno de un proceso productivo informacional y fragmentado, cuando ésta se ve atravesada por conflictos intrasindicales y reclamos de reencuadramiento gremial, originados en situaciones de tercerización y de subcontratación laboral.

Asimismo, hemos identificado un segundo grupo de acciones concretas de manifestación y de protesta que estuvieron lideradas orgánicamente por una organización sindical, para visibilizar la condición de vulnerabilidad de los trabajadores de las empresas comercializadoras de servicios de teleatención y ampliar sus márgenes de negociación. Nos referimos al intento de revertir las dificultades regulatorias que conlleva la tercerización de los servicios de teleatención, en términos normativos amplios, con una propuesta legislativa como la Ley "Estatuto para los Teleoperadores de Centros de Atención de Llamadas (Call Centers)", del año 20137. Esta estrategia fue impulsada activamente, desde su primera presentación en el año 2005, por Sindicato Telefónico de Buenos Aires.

A partir de la definición amplia de la categoría laboral de "teleoperador", (artículo 2. referido al Ámbito de Aplicación Personal, donde se considera como "teleoperador" "a toda persona que desempeñe tareas de atención, recepción o emisión de conexiones telefónicas o telemáticas", ya sea para "recepción de reclamos o denuncias; recepción de solicitudes de información o asesoramiento sobre aspectos técnicos, comerciales o administrativos; venta y promoción de productos o servicios; contactos de fidelización de clientes; realización de encuestas e investigaciones de mercadeo"), el Proyecto de Ley establece garantías en relación con el tipo de contratación, los salarios, la extensión de la jornada laboral (jornada laboral de seis horas) y las condiciones ambientales apropiadas para el desarrollo del trabajo de teleatención. En este sentido, su alcance no se limita sólo a los centros de llamadas que pertenecen a las empresas telefónicas, sino que incluye también a aquellos gestionados por las empresas comercializadoras de servicios (tercerizadoras) y a los centros de llamadas internos de empresas de distintos sectores (telecomunicaciones, finanzas, seguros, recreación, turismo, hostelería, etc.).

De aprobarse alguna vez dicho Proyecto, miles de trabajadores, mayoritariamente empleados de empresas tercerizadoras de servicios empresariales, mejorarían sus condiciones laborales, salariales, y las normas de seguridad e higiene que rigen la actividad.

7. El Proyecto de Ley (5047-D-2013) fue presentado el 02/07/2013. Documento disponible en: www. diputados.gov.ar. Como antecedente se puede citar Proyecto de Ley, "Régimen especial básico del teleoperador" (108/4676 -D- 05). Disponible en: http://www.leydelteleoperador.com.ar/ Sin tratamiento efectivo, y el "Proyecto de Ley de "Protección del Teleoperador" (expediente número: 3557/10). Disponible en : www.senado.gov.ar 
Sin embargo, la iniciativa ha sido presentada sucesivas veces desde 2005, perdiendo en todos los casos estado parlamentario. La presión ejercida por las empresas nucleadas en la Cámara Argentina de Centros de Contacto para frenar la iniciativa, anunciando una inminente deslocalización de actividades en caso de aprobación, influyó en el curso de los acontecimientos.

A partir de 2008, cuando la actividad de los centros de llamadas dejó de crecer debido a la caída registrada en las operaciones offshore, junto con la pérdida de empleos, los trabajadores también vieron disminuida su fuerza para seguir presionando por el mejoramiento de sus condiciones laborales. Como hemos mencionado en los apartados anteriores, las empresas comercializadoras de servicios de teleatención respondieron a la reorientación de la actividad profundizando, efectivamente, el proceso de relocalición de centros de llamadas en el interior del país y deslocalizando las operaciones offshore en otros países de la región con mejores costos que la Argentina.-

Durante 2006, se formó otra agrupación que también disputó la representación de los trabajadores del sector, nos referimos a la ATCCAC, la Asociación de Trabajadores de Centros de Contactos y Afines de Córdoba ${ }^{8}$. Esta organización surgió a partir de un conflicto puntual en una de las empresas más grandes del sector, Jazzplat S.A. (subsidiaria de la española Jazztel S.A), que se desencadenó a partir del despido masivo de un centenar de trabajadores, tras la decisión de deslocalizar operaciones. A partir de ese conflicto los trabajadores comenzaron a gestar una organización que se fijó como objetivo prioritario el reconocimiento gremial. En este sentido, lo que representa una diferencia cualitativa, comparando el rol de la ATCCAC con el de los otros grupos que aspiraron en algún momento a organizar a los trabajadores de los centros de llamadas, es que la ATCCAC obtuvo en febrero de 2007 la inscripción en el Registro de Asociaciones sindicales con carácter de asociación gremial de primer grado, para agrupar a los trabajadores que prestaban servicios en relación de dependencia con empresas comercializadoras de servicios de teleatención con zona de actuación en la ciudad de Córdoba (resolución MTEySS no 151-2007).

A partir del momento en que se consiguió la inscripción gremial se comenzó con un trabajo muy intenso de expansión mediante afiliaciones (cerca de seis mil quinientos afiliados en dos años) para sortear la política antisindical de las empresas del sector y el temor de los trabajadores a afiliarse'. La meta final era llegar a negociar un convenio

8. Entrevista al Secretario Gremial de ATCCAC, realizada en Córdoba, mayo de 2010.

9. Según se expresa en la entrevista al Secretario Gremial de ATCCAC: "Nosotros tenemos una forma de afiliar que nos ha dado resultados, en el sentido de que los trabajadores de los calls centers tienen mucho miedo con la afiliación, ¿si?, muchos por miedo no porque no tengan ganas, sino que tienen miedo a las represalias empresarias, ¿isi?, eh (...) entonces hacemos un "delivery", vamos con la ficha y se la hacemos firmar en domicilio, para que no tenga el miedo de que lo estamos afiliando dentro de la empresa. Siempre 
colectivo de trabajo que habilitase mejores condiciones salariales y de trabajo de los agentes telefónicos. Siete años después, esta agrupación obtuvo la personería gremial, como Asociación de Trabajadores Argentinos de Centros de Contacto (ATACC), y negoció por primera vez un convenio colectivo de trabajo, -CCT 688/14-, fuera de la órbita del sindicato de Comercio, para los centros de llamadas de la provincia de Córdoba. El convenio colectivo 688/14 de la ATACC con la Cámara Argentina de Centros de Contacto, ha ido extendiendo su ámbito de aplicación, en noviembre de 2015, entró en vigencia en las empresas que nuclea la Cámara en las provincias argentinas de Chaco, Tucumán, Salta, Mendoza, San Luis y en Ciudad Autónoma de Buenos Aires.

En relación con las modalidades y prácticas de movilización de la ATCCAC, desde 2006 se llevaron adelante un abanico de acciones de diversa índole (Del Bono, Bulloni, 2013). Entre éstas, activa presencia en los medios locales de comunicación denunciando las malas condiciones de trabajo; asesoramiento y apoyo legal a trabajadores en los reclamos y juicios laborales; trabajo en conjunto con profesionales de la salud para el tratamiento y prevención de enfermedades laborales propias de los agentes telefónicos, y activa participación en movilizaciones de trabajadores en rechazo del encuadramiento sindical en Comercio, por suba de salarios y por mejora de las condiciones laborales ${ }^{10}$.

Ahora bien, si bien las condiciones laborales que se regulan en el convenio colectivo 688/14 de la ATACC reconocen la especificidad de la actividad de trabajo de los agentes telefónicos y los riesgos y problemas laborales que plantea la actividad, según nuestro análisis, al comparar cómo se fijan las condiciones relativas a la jornada y al salario de los trabajadores de los centros de llamadas respecto del convenio colectivo 130/75 del sindicato de Comercio, no se advierten diferencias sustanciales entre ambos acuerdos. Según lo establecido para mediados de 2015, para los trabajadores que se encuentran bajo la órbita del convenio de comercio (CCT N 130/75) se dispone una jornada de 36 horas semanales y un salario equivalente al escalafón de Administrativo B, proporcional de U\$S 713 (el salario básico por ocho horas de trabajo equivalía a de U\$S 951), para los trabajadores de la ATACC (CCT 688/14) la jornada semanal también es de 36 horas y el salario es levemente superior, ascendiendo a U\$S 749 (con una diferencia menor de un

digo, no afilien dentro de la empresa sino afuera, cuando tenés una reunión, cuando te juntás con tus amigos el fin de semana, te juntás a comer una pizza con los compañeros de trabajo, y ahí se habla de lo sindical, se habla de lo que es el armado de las fichas, lo que es la afiliación, y bueno así vamos trabajando y hemos tenido muy bueno resultados".

10. Se trata de dos marchas que tuvieron lugar en mayo y junio de 2009. La primera habría sido impulsada por ATCCAC y fue más masiva, y la segunda fue organizada en forma conjunta con otras agrupaciones -La Coordinadora y Calls en Lucha-. Tal como se aprecia en los sitios web, como así también en los relatos de los referentes entrevistados, estas marchas fueron muy significativas para consolidar la identidad de estos grupos (ver imágenes en: $\underline{h t t p: / / w w w . a t c c a c . o r g / ~ y ~ h t t p: / / w w w . c a l l s e n l u c h a . b l o g s p o t . c o m /) . ~}$ 
$5 \%)^{11}$. Es importante observar que la jornada de trabajo de seis horas diarias y 36 horas semanales es en términos legales la jornada normal y habitual de los centros de llamadas, debido a las características estresantes de la actividad, y que las empresas tercerizadoras deberían abonar el salario convencional previsto para una jornada normal de trabajo de ocho horas. Sin embargo, esto no es lo que se establece en ninguno de los dos convenios colectivos, que fijan descuentos proporcionales en relación con la jornada de ocho horas.

A modo de síntesis, podemos señalar que, como fruto de los avances en el plano institucional de la negociación colectiva, la nueva representación gremial de los trabajadores de los centros de llamadas logró aprobar un nuevo convenio colectivo de trabajo que refleja mejor la naturaleza específica de la actividad de los agentes telefónicos pero que no es suficiente para combatir la flexibilización laboral ya que, en los hechos, las empresas que gestionan los servicios de teleatención consiguieron imponer sus pautas de flexibilización. Como acabamos de describir, en el plano concreto de la relación laboral, la regulación ejercida por la acción sindical presenta limitaciones que parecen difíciles de revertir. La lógica de reducción de costos que caracteriza a los centros de llamadas, sensiblemente agravada por la fluctuación de las relocalizaciones y las deslocalizaciones internacionales de los últimos años, tiñe los horizontes de trabajo con una marcada incertidumbre frente a la cual los sindicatos y los propios trabajadores tienden a resignar derechos laborales.

\section{CONCLUSIONES}

Hemos comenzado este trabajo señalando que el ingreso en una etapa de tercerización y deslocalización global ha dotado a la economía internacional de un nuevo nivel de fluidez y que ello tiene implicancias significativas sobre el trabajo y sus modalidades de regulación. Planteamos, también, la importancia que adquiere actualmente la prestación de servicios empresariales a distancia a partir de las exigencias de la producción descentralizada -pero crecientemente integrada- del capitalismo actual.

En el marco de estas tendencias, buscamos evidenciar que el fenómeno de las deslocalizaciones internacionales no implica para los trabajadores y sus organizaciones gremiales un juego de suma cero, sino un gran desafío, que impone al actor sindical la necesidad de desarrollar estrategias para avanzar en el proceso de protección de derechos laborales en un contexto productivo cambiante, amenazado por el problema de la deslocalización.

A partir de un caso concreto de estudio hemos visto que, para la Argentina, la principal ventaja que ha traído la enorme expansión de los centros de llamadas y de las empresas

11. Se calculó el valor en dólares americanos en base a un tipo de cambio de $1 \mathrm{U} \$ \mathrm{~S}=9,075$ pesos argentinos (fecha 27/7/2015). Convenio Colectivo de Trabajo CCT 668/14 y Modificaciones: $\underline{\text { http://www.atacc.com. }}$ ar/index.php/info-legal. 
comercializadoras de servicios empresariales, -estimulada inicialmente por su inserción en encadenamientos globales de exportación de servicios-, ha sido la creación de un buen número de empleos que son mayormente registrados y formales. El crecimiento de la actividad de los centros de llamadas, intensiva en mano de obra, ha permitido el ingreso al mercado laboral de miles de jóvenes y allí reside su fuente de legitimación más importante. Sin embargo, también señalamos que este impacto positivo queda relativizado por la imposibilidad que muestra la actividad para ofrecer trabajos de calidad, carrera profesional, buenos salarios y algún tipo de formación para sus trabajadores.Al respecto, hemos argumentado que tal situación se explica, en parte, tomando en consideración el tipo de organización y de gestión de la fuerza de trabajo que existe en los centros de llamadas y analizando cómo estas dimensiones están determinadas por las estrategias de flexibilización laboral y de intensificación del trabajo que son el motor de esta actividad económica globalizada.

Asimismo, completamos dicha explicación a partir del análisis de las dificultades que plantea para la organización sindical y de los propios trabajadores un proceso productivo muy deslocalizado y que cuenta con un soporte tecnológico que relativiza la eficacia de la organización sindical clásica. Llegado el caso, cuando los agentes telefónicos deciden organizarse, los servicios pueden reencauzarse fácilmente hacia otros centros de llamadas, dejando a los trabajadores, desarticulados. En este sentido, la actividad no está condicionada por el espacio físico particular ya que es posible organizar una red de centros de llamadas que actúen como soporte de la continuidad del flujo de trabajo. En el caso analizado, con la apertura de nuevos centros de trabajo en el interior del país y con las deslocalizaciones de servicios en otros países de América Latina (Colombia, Perú, y actualmente Centroamérica), se consiguió neutralizar protestas y reclamos sindicales por mejores condiciones salariales y de trabajo. Hemos analizado, entonces, las limitaciones que presenta el accionar sindical en defensa de los trabajadores que se ven abarcados por ese proceso de deslocalización.

En este sentido, se torna cada vez más evidente para los sindicatos que, ante las permanentes reestructuraciones empresarias, la fuerza de trabajo solo podrá recuperar poder de negociación a través de una mayor unidad sectorialy respaldándose en estrategias abarcativas que desborden las problemáticas de cada empresa particular e incluso que traspasen lo local (Taylor y Bain, 2008). En los centros de llanadas offshore, impedir la pérdida de empleos que deviene como resultado de las permanentes deslocalizaciones, y a su vez mejorar la calidad del trabajo, resulta todo un desafío en un contexto donde la lógica de maximización de ganancias y de reducción de costos aceleran la fragmentación y relocalización de los procesos productivos a la vez que debilitan la capacidad de negociación de los trabajadores y de sus representantes gremiales.

En síntesis, podemos concluir que en el caso analizado, existe una relativa insuficiencia 
de las respuestas nacionales, habida cuenta del contexto que impone la globalización y la deslocalización de actividades; en este sentido, si bien las dinámicas nacionales son necesarias para mitigar los efectos negativos de dichos procesos, se precisan fuertes regulaciones adicionales, también configuradas en ámbitos internacionales (Ermida Uriarte, 2009). Es decir, se requiere de un redimensionamiento del accionar sindical que traspase las fronteras nacionales (Boix, 2007). 


\section{BIBLIOGRAFÍA}

Abal Medina, Paula (coord..) (2009), "Senderos bifurcados. Prácticas sindicales en tiempos de precarización sindical”. Buenos Aires: Ed. Prometeo.

Boix, Isidor (2007) “¿Es necesario y el sindicato "global”? Gaceta Sindical: Reflexión y Debate, No. 8, 2007, pp. 113-133.

De la Cámara Arilla, Carmen y Puig Gómez, Albert (2007) “Deslocalizaciones: ¿mito o realidad? El caso español frente a los países de europa central y oriental”. Revista de Economía Mundial 16. Barcelona: Universidad Autónoma, pp. 169-193.

Del Bono, Andrea y Bulloni, María Noel. (2008), "Experiencias laborales juveniles. Los agentes telefónicos de call centers offshore en Argentina”. Trabajo y Sociedad, Número 10, vol. IX, pp. 1-21.

Del Bono, Andrea y Henry, Laura. (2008), "La acción sindical en el marco de las formas globalizadas de producción. Reflexiones a partir de la expansión de los call centers de servicios para exportación en Argentina”. Revista Latinoamericana de Estudios del Trabajo II Época, Año 13, Número 20, pp. 7-31.

Del Bono, A.; Gorjup, M.; Valverde, M.; Henry, M. (2013), "Call centres employment practices in glogal value networks: A view fron Argentina as a receiving economy", Economic and Industrial Democracy, 34(4), pp. 693-717.

Del Bono, Andrea y Bulloni María Noel (2013), "Los claroscuros de la revitalización sindical en contextos de tercerización: un apunte regional sobre el sector de call centers en Argentina”, en Senén González C. y Del Bono. A. (dir.), La revitalización sindical en la Argentina: alcances y perspectivas. Buenos Aires: Prometeo, pp. 128-150.

Ermida Uriarte, Oscar (2007) “Deslocalización, globalización y derechos del trabajo". IUSLabor 1/2007, pp.1-17 (Reconstrucción de la conferencia dictada el 17 de noviembre de 2005 en la Jornada sobre "Los problemas laborales de las deslocalizaciones productivas", organizada por la Consejería de trabajo e industria de la Generalitat de Cataluña).

Ermida Uriarte, Oscar (2009) "La política laboral de los gobiernos progresistas, el posneoliberalismo y el movimiento sindical”. En Guillermo Martner (coord.,) Movimiento sindical gobiernos progresistas: un primer balance en la región. Montevideo: Fundación Ebert.

Figari Claudia, et al. (coord.) (2010), El movimiento obrero en disputa. La organización colectiva de los trabajadores, su lucha y resistencia en la Argentina del siglo XX, Buenos Aires: Ciccus/Ceil.

Flores Sánchez, Gabriel (2010) "La acción sindical y las dinámicas de deslocalización internacional". Cuadernos de Relaciones Laborales. Vol. 28 Num. 1, pp. 65-86 
Harvey, David (1998) La condición de la posmodernidad, investigación sobre los orígenes del cambio cultural. Buenos Aires: Amorrortu editores.

Holman, David; Batt, Rosemary; Holtgrewe, Ursula (2007): The Global Call Center Report: International Perpectives on Managmente and Employment (Executive summary) [Electronic version]. Ithaca, NY: Authors.

Knorringa Peter y Pegler Lee (2006) Globalisation, firm upgrading and impacts on labour. Tijdschrift voor Economische en Sociale Geografie 97(5), pp. 470-479.

Lenguita, Paula (2011), "Revitalización desde las bases del sindicalismo argentino". Nueva Sociedad. Buenos Aires: Friedrich Ebert. vol. n²32, pp. 137-149.

Luengo, Fernando (2010) "Las deslocalizaciones Internacionales. Una visión desde la economía crítica”. Cuadernos de Relaciones Laborales. Vol. 28 pp. 87-130

Miguelez, Fausto (2008) "Globalización y sindicalismo. Presentación". Cuadernos de Relaciones Laborales. Vol. 26, núm. 1, pp. 9-16.

Moench, Erick (2011), «Inversiones en servicios: Nuevas territorialidades en la expansión de call centers en el interior de la Argentina, 2002-2010 ». Revista de estudios regionales y mercado de trabajo, (7), pp. 59-88.

Murillo, Victoria ( 1997) "La adaptación del sindicalismo argentino a las reformas de mercado en la primera presidencia de Menem". Desarrollo Económico, Vol. 37, № 147, pp. 419-446.

Novick, Marta (2001) "Nuevas reglas de juego en la Argentina, competitividad y actores sindicales". En Enrique de la Garza Toledo (comp.) Los sindicatos frente a los procesos de transición política. Buenos Aires: CLACSO, pp. 25.46.

Palomino, Héctor (2007) "La instalación de un nuevo régimen de empleo en Argentina." Ponencia presentada en el $8^{\circ}$ Congreso de ASET, Buenos Aires, Aset.pp. 1-30.

Palomino, Héctor y Trajtemberg, David (2006), "Una nueva dinámica de las relaciones laborales y la negociación colectiva en la Argentina”. Revista de trabajo. Buenos Aires, Ministerio de Trabajo, Empleo y Seguridad Social, Año 2, No3, pp. 47-68

Peck, Jamie (1996) Work-Place. The social Regulation of Labor Markets. New York: The Guildford Press.

Piña, Joaquín, (2005). La deslocalización de funciones no esenciales en las empresas: Oportunidades Para Exportar Servicios. El Caso De Chile. Chile: CEPAL.

Sassen, Saskia (2003), Los espectros de la globalización. Buenos Aires-México: Fondo de Cultura Económica.

Sassen, Saskia (2007), Una sociología de la globalización. Buenos Aires: Katz Editores. 
Senén González, Cecilia y Garro Silvia (2008) "Fortaleza colectiva y fragmentación sindical en el sector de tele-comunicaciones en la Argentina post-devaluación". Revista Latinoamericana de Estudios del Trabajo. Año 13, numero 19, pp. 30-47.

Senén González, Cecilia y Haidar, Julieta (2009), "Los debates acerca de la "revitalización sindical" y su aplicación en el análisis sectorial en Argentina". Revista Latinoamericana de Estudios del Trabajo, II, Venezuela, Época, Año 22, Número 22, 5-32.

Taylor, Peter y Bain, Phil (2008) "No passage to India? Initial responses of UK trade unions to call centre offshoring”. Industrial Relations Journal. Vol. 39 (1), pp. 5-23.

Trajtemberg, David (et.al) (2009), "Los determinantes de la negociación colectiva en la Argentina. Debates teóricos y evidencias empíricas”. Serie Estudios: Trabajo, Ocupación y Empleo $N^{\circ}$ 9, Buenos Aires, Subsecretaría de Programación Técnica y Estudios Laborales, MTEySS, pp. 13-35.

Walter, Jorge y Senén González, Cecilia (2000), La privatización de las telecomunicaciones en América Latina. Estudio comparativo. Buenos Aires: CEPAL-(ECLAC, CLA-98-1215).

\section{FUENTES}

ATKearney, (2007). 'Global Services Location Index 2007', Offshoring for Long-Term Advantage. Chicago: ATKearney.

Camara Argentina de Centros de Contacto (2013), Dossier de Prensa, CACC, Buenos Aires.

Convergencia Research (2008) Contact Centers 2007. Buenos Aires, Argentina.

Frost and Sullivan (2008) Latin America contact centre outsourcing services markets. https://store.frost.com/ [Consultado el 5 mayo de 2008].

Prom-Perú (2015) Informe especializado: Call centers en Centroamérica. http://www. siicex.gob.pe/siicex/resources/estudio/590987468rad2E491.pdf [Consultado el 2 de abril de 2015]. 